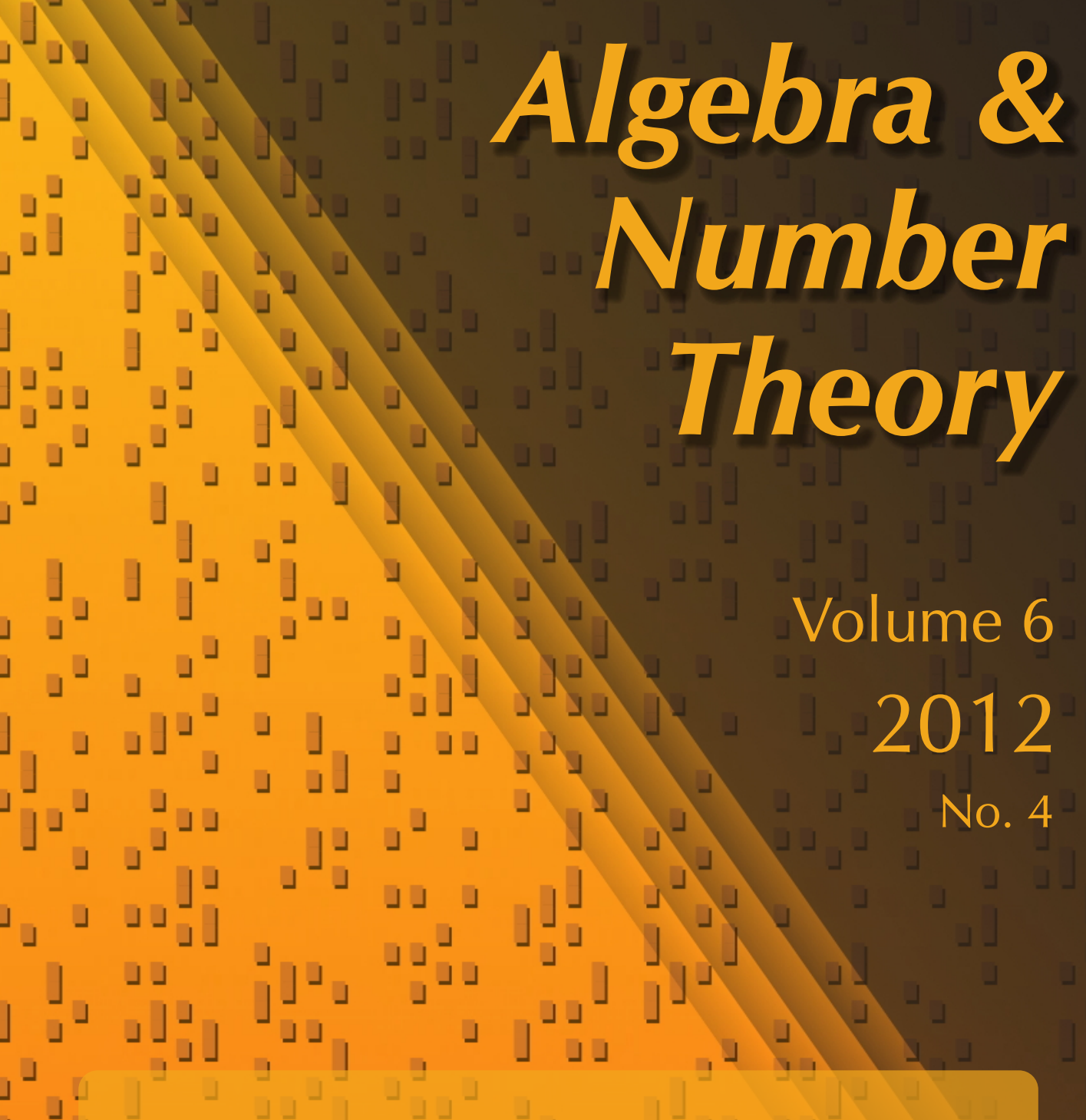

Nonuniruledness results for spaces of rational curves
in hypersurfaces

Nonuniruledness results for spaces of rational curves
in hypersurfaces

Nonuniruledness results for spaces of rational curves
in hypersurfaces 


\title{
Nonuniruledness results for spaces of rational curves in hypersurfaces
}

\author{
Roya Beheshti
}

\begin{abstract}
We prove that the sweeping components of the space of smooth rational curves in a smooth hypersurface of degree $d$ in $\mathbb{P}^{n}$ are not uniruled if $(n+1) / 2 \leq d \leq n-3$. We also show that for any $e \geq 1$, the space of smooth rational curves of degree $e$ in a general hypersurface of degree $d$ in $\mathbb{P}^{n}$ is not uniruled roughly when $d \geq e \sqrt{n}$.
\end{abstract}

\section{Introduction}

Throughout this paper, we work over an algebraically closed field $k$ of characteristic zero. Let $X$ be a smooth hypersurface of degree $d$ in $\mathbb{P}^{n}$, and for $e \geq 1$, let $R_{e}(X)$ denote the closure of the open subscheme of $\operatorname{Hilb}_{e t+1}(X)$ parametrizing smooth rational curves of degree $e$ in $X$. It is known that if $d<(n+1) / 2$ and $X$ is general, then $R_{e}(X)$ is an irreducible variety of dimension $e(n+1-d)+n-4$, and it is conjectured that the same holds for general Fano hypersurfaces; see [Harris et al. 2004; Coskun and Starr 2009]. If $X$ is not general, $R_{e}(X)$ may be reducible. We call an irreducible component $R$ of $R_{e}(X)$ a sweeping component if the curves parametrized by its points sweep out $X$, or equivalently, if for a general curve $C$ parametrized by $R$ the normal bundle of $C$ in $X$ is globally generated. If $d \leq n-1$, or if $d=n$ and $e \geq 2$, then $R_{e}(X)$ has at least one sweeping component.

In this paper, we study the birational geometry of sweeping components of $R_{e}(X)$. Recall that a projective variety $Y$ of dimension $m$ is called uniruled if there is a variety $Z$ of dimension $m-1$ and a dominant rational map $Z \times \mathbb{P}^{1} \rightarrow Y$. We are interested in the following question: for which values of $n, d$, and $e$ does $R_{e}(X)$ have nonuniruled sweeping components? Our original motivation for this study comes from the question of whether or not general Fano hypersurfaces of low indices are unirational.

We give a complete answer to the above question when $(n+1) / 2 \leq d \leq n-3$ :

Theorem 1.1. Let $X$ be any smooth hypersurface of degree $d$ in $\mathbb{P}^{n}$, where $(n+1) / 2 \leq d \leq n-3$. Then for all $e \geq 1$, no sweeping component of $R_{e}(X)$ is uniruled.

MSC2010: primary 14J70; secondary 14J40, 14E05.

Keywords: rational curves on hypersurfaces. 
We also consider the case $d=n-2$ and prove:

Theorem 1.2. Let $X$ be a smooth hypersurface of degree $n-2$ in $\mathbb{P}^{n}$, and let $C$ be a smooth rational curve of degree $e$ in $X$. Every irreducible sweeping component of $R_{e}(X)$ which contains $C$ is nonuniruled provided that when we split the normal bundle of $C$ in $\mathbb{P}^{n}$ as a sum of line bundles

$$
N_{C / \mathbb{P}^{n}}=\mathrm{O}_{C}\left(a_{1}\right) \oplus \cdots \oplus \mathcal{O}_{C}\left(a_{n-1}\right),
$$

we have $a_{i}+a_{j}<3$ for every $1 \leq i<j \leq n-1$.

When $n=5$ and $d=3, R_{e}(X)$ is irreducible for any smooth $X$; see [Coskun and Starr 2009]. J. de Jong and J. Starr [2004] studied the birational geometry of $R_{e}(X)$ with regards to the question of rationality of general cubic fourfolds. Let $\bar{M}_{0,0}(X, e)$ be the Kontsevich moduli stack of stable maps of degree $e$ from curves of genus zero to $X$ and $\bar{M}_{0,0}(X, e)$ the corresponding coarse moduli scheme. There is an open subscheme of $\bar{M}_{0,0}(X, e)$ parametrizing smooth rational curves of degree $e$ in $X$. Presenting a general method to produce differential forms on desingularizations of $\bar{M}_{0,0}(X, e)$, de Jong and Starr prove that if $X$ is a general cubic fourfold, then $R_{e}(X)$ is not uniruled when $e>5$ is an odd integer, and the general fibers of the MRC fibration of a desingularization of $R_{e}(X)$ are at most 1-dimensional when $e>4$ is an even integer.

If $X$ is a general cubic fourfold, then for a general rational curve $C$ of degree $e$ in $X$, the normal bundle of $C$ in $\mathbb{P}^{5}$ is isomorphic to $\mathscr{O}_{C}((3 e-1) / 2)^{\oplus 4}$ if $e \geq 5$ is odd and to $\mathscr{O}_{C}(3 e / 2)^{\oplus 2} \oplus \mathscr{O}_{C}((3 e / 2)-1)^{\oplus 2}$ if $e \geq 6$ is an even integer; see [de Jong and Starr 2004, Proposition 7.1]. Thus Theorem 1.2 gives a new proof of the result of de Jong and Starr when $e \geq 5$ is odd. In Section 4 we study the case when $e$ is an even integer and show:

Theorem 1.3. Let $X$ be a smooth cubic fourfold, and let $C$ be a general smooth rational curve of degree $e \geq 5$ in $X$.

- $R_{e}(X)$ is not uniruled if $e$ is odd and $N_{C / \mathbb{P}^{5}}=\mathrm{O}_{C}((3 e-1) / 2)^{\oplus 4}$.

- If $\widetilde{R}$ is a desingularization of $R_{e}(X)$, then the general fibers of the MRC fibration of $\widetilde{R}$ are at most 1-dimensional if e is even and

$$
N_{C / \mathbb{P}^{5}}=\mathrm{O}_{C}(3 e / 2)^{\oplus 2} \oplus \mathcal{O}_{C}((3 e / 2)-1)^{\oplus 2} .
$$

It is an interesting question whether or not the splitting type of $N_{C / \mathbb{P}^{n}}$ is always as above for a general rational curve $C$ of degree $\geq 5$ in an arbitrary smooth cubic fourfold.

Finally, we consider the case $d<(n+1) / 2$. When $d^{2} \leq n, R_{e}(X)$ is uniruled. In fact, in this range a much stronger statement holds: for every $e \geq 2$, the space of based, 2-pointed rational curves of degree $e$ in $X$ is rationally connected in a suitable sense; see [de Jong and Starr 2006; Starr 2006]. By [Harris et al. 2004], 
when $X$ is general and $d<(n+1) / 2, \bar{M}_{0,0}(X, e)$ is irreducible and therefore it is birational to $R_{e}(X)$. Starr [2003] shows that if $d<\min (n-6,(n+1) / 2)$ and $d^{2}+d \geq 2 n+2$, then for every $e \geq 1$, the canonical divisor of $\bar{M}_{0,0}(X, e)$ is big. This suggests that when $d^{2}+d \geq 2 n+2$ and $X$ is general, $R_{e}(X)$ may be nonuniruled. In Section 5, we show:

Theorem 1.4. Let $X \subset \mathbb{P}^{n}(n \geq 12)$ be a general hypersurface of degree $d$, and let $m \geq 1$ be an integer. If a general smooth rational curve $C$ in $X$ of degree $e$ is $m$-normal (that is, if the global sections of $\mathcal{O}_{\mathbb{p} n}(m)$ map surjectively to those of $\left.\left.\mathrm{O}_{\mathbb{p} n}(m)\right|_{C}\right)$, and if

$$
d^{2}+(2 m+1) d \geq(m+1)(m+2) n+2,
$$

then $R_{e}(X)$ is not uniruled.

In particular, since every smooth curve of degree $e \geq 3$ in $\mathbb{P}^{n}$ is $(e-2)$-normal, it follows that $R_{e}(X)$ is not uniruled when $X$ is general and

$$
d^{2}+(2 e-3) d \geq e(e-1) n+2 .
$$

\section{A consequence of uniruledness}

In this section, we prove a proposition, analogous to the existence of free rational curves on nonsingular uniruled varieties, for varieties whose spaces of smooth rational curves are uniruled. We first fix notation and recall some definitions.

For a morphism $f: Y \rightarrow X$ between smooth varieties, by the normal sheaf of $f$ we will mean the cokernel of the induced map on the tangent bundles $T_{Y} \rightarrow f^{*} T_{X}$.

If $Y$ is an irreducible projective variety, and if $\widetilde{Y}$ is a desingularization of $Y$, then the maximal rationally connected (MRC) fibration of $\widetilde{Y}$ is a smooth morphism $\pi: Y^{0} \rightarrow Z$ from an open subset $Y^{0} \subset \widetilde{Y}$ such that the fibers of $\pi$ are all rationally connected, and such that for a very general point $z \in Z$, any rational curve in $\widetilde{Y}$ intersecting $\pi^{-1}(z)$ is contained in $\pi^{-1}(z)$. The MRC fibration of any smooth variety exists and is unique up to birational equivalences [Kollár et al. 1992].

Let $Y$ be an irreducible projective variety, and assume the fiber of the MRC fibration of $\widetilde{Y}$ at a general point is $m$-dimensional. Then it follows from the definition that there is an irreducible component $Z$ of $\operatorname{Hom}\left(\mathbb{P}^{1}, Y\right)$ such that the map $\mu_{1}: Z \times \mathbb{P}^{1} \rightarrow Y$ defined by $\mu_{1}([g], b)=g(b)$ is dominant and the image of the map $\mu_{2}: Z \times \mathbb{P}^{1} \times \mathbb{P}^{1} \rightarrow Y \times Y$ defined by $\mu_{2}\left([g], b_{1}, b_{2}\right)=\left(g\left(b_{1}\right), g\left(b_{2}\right)\right)$ has dimension $\geq \operatorname{dim} Y+m$.

Proposition 2.1. Let $X \subset \mathbb{P}^{n}$ be a nonsingular projective variety. If an irreducible sweeping component $R$ of $R_{e}(X)$ is uniruled, then there exist a smooth rational surface $S$ with a dominant morphism $\pi: S \rightarrow \mathbb{P}^{1}$ and a generically finite morphism $f: S \rightarrow X$ with the following two properties: 
(i) If $C$ is a general fiber of $\pi$, then $\left.f\right|_{C}$ is a closed immersion onto a smooth curve parametrized by a general point of $R$.

(ii) If $N_{f}$ denotes the normal sheaf of $f$, then $\pi_{*} N_{f}$ is globally generated.

Moreover, if the fiber of the MRC fibration of a desingularization of $R$ at a general point is at least $m$-dimensional, then there are such $S$ and $f$ with the additional property that $\pi_{*} N_{f}$ has an ample subsheaf of rank $=m-1$.

Proof. Let $U \subset R \times X$ be the universal family over $R$. Since $R$ is uniruled, there exist a quasiprojective variety $Z$ and a dominant morphism $\mu: Z \times \mathbb{P}^{1} \rightarrow R$. Let $V \subset Z \times \mathbb{P}^{1} \times X$ be the pullback of the universal family to $Z \times \mathbb{P}^{1}$, and denote by $q: V \rightarrow Z \times X$ and $p: V \rightarrow Z$ the projection maps.

Consider a desingularization $g: \widetilde{V} \rightarrow V$, and let $\tilde{q}=q \circ g$ and $\tilde{p}=p \circ g$. Let $z \in Z$ be a general point, and denote the fibers of $p$ and $\tilde{p}$ over $z$ by $S$ and $\widetilde{S}$, respectively. Let $f: S \rightarrow X$ be the restriction of $q$ to $S$, and let $\tilde{f}=f \circ g: \widetilde{S} \rightarrow X$. Since $z$ is general, by generic smoothness $\widetilde{S}$ is a smooth surface whose general fiber over $\mathbb{P}^{1}$ is a smooth connected rational curve. We claim that $\widetilde{S}$ and $\tilde{f}$ satisfy the desired properties. The first property is clearly satisfied.

Since every coherent sheaf on $\mathbb{P}^{1}$ splits as a torsion sheaf and a direct sum of line bundles, to show that $\pi_{*} N_{f}$ is globally generated it suffices to check that the restriction map $\left.H^{0}\left(\mathbb{P}^{1}, \pi^{*} N_{f}\right) \rightarrow N_{f}\right|_{b}$ is surjective for a general point $b \in \mathbb{P}^{1}$, or equivalently, that the restriction map $H^{0}\left(S, N_{f}\right) \rightarrow H^{0}\left(C,\left.N_{f}\right|_{C}\right)$ is surjective for a general fiber $C$. To show this, we consider the Kodaira-Spencer map associated to $\widetilde{V}$ at a general point $z \in Z$. Denote by $N_{\tilde{q}}$ the normal sheaf of the map $\tilde{q}$. We get a sequence of maps

$$
T_{Z, z} \rightarrow H^{0}\left(\widetilde{S}, \tilde{p}^{*} T_{Z} \mid \widetilde{S}\right) \rightarrow H^{0}\left(\widetilde{S}, \tilde{q}^{*} T_{X \times Z} \mid \widetilde{S}\right) \rightarrow H^{0}\left(\widetilde{S}, N_{\tilde{q}} \mid \widetilde{S}\right) .
$$

Let $b$ be a general point of $\mathbb{P}^{1}$. Composing the above map with the projection map $T_{Z \times \mathbb{P}^{1},(z, b)} \rightarrow T_{Z, z}$, we get a map $T_{Z \times \mathbb{P}^{1},(z, b)} \rightarrow H^{0}\left(\widetilde{S},\left.N_{\tilde{q}}\right|_{\tilde{S}}\right)$. Note that if $N_{\tilde{f}}$ denotes the normal sheaf of $\tilde{f}$, then $\left.N_{\tilde{q}}\right|_{\tilde{S}}$ is naturally isomorphic to $N_{\tilde{f}}$. Also, if $C$ is the fiber of $\pi: \widetilde{S} \rightarrow \mathbb{P}^{1}$ over $b$, then since $b$ is general, $C$ is smooth, and we have a short exact sequence

$$
\left.0 \rightarrow N_{C / \tilde{S}} \rightarrow N_{\tilde{f}(C) / X} \rightarrow N_{\tilde{f}}\right|_{C} \rightarrow 0 .
$$

So we get a commutative diagram

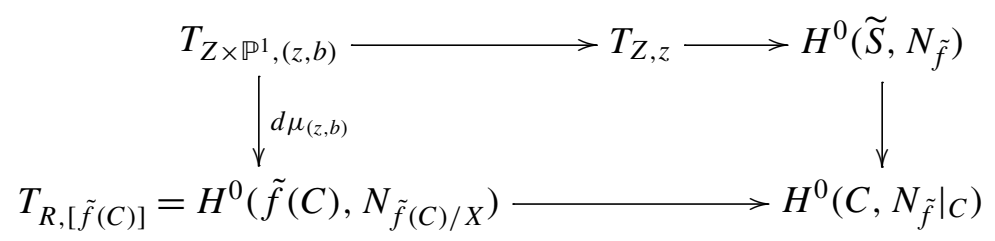


Since $\mu$ is dominant, and since $R$ is sweeping and therefore generically smooth, $d \mu_{(z, b)}$ is surjective. Since the bottom row is also surjective, the map $H^{0}\left(\widetilde{S}, N_{\tilde{f}}\right) \rightarrow$ $H^{0}\left(C,\left.N_{\tilde{f}}\right|_{C}\right)$ is surjective as well. Thus $\tilde{\pi}_{*} N_{\tilde{f}}$ is globally generated.

Suppose now that $R$ is uniruled and that the general fibers of the MRC fibration of $R$ are at least $m$-dimensional. Let $\operatorname{dim} R=r$. Then there exists a morphism $\mu_{1}: Z \times \mathbb{P}^{1} \rightarrow R$ such that the image of

$$
\mu_{2}: Z \times \mathbb{P}^{1} \times \mathbb{P}^{1} \rightarrow R \times R, \quad \mu_{2}\left(z, b_{1}, b_{2}\right)=\left(\mu_{1}\left(z, b_{1}\right), \mu_{1}\left(z, b_{2}\right)\right)
$$

has dimension $\geq r+m$. Constructing $\widetilde{S}$ and $\tilde{f}$ as before, and if $C_{1}$ and $C_{2}$ denote the fibers of $\pi$ over general points $b_{1}$ and $b_{2}$ of $\mathbb{P}^{1}$, then the image of the map

$$
\begin{aligned}
d \mu_{2}: T_{Z \times \mathbb{P}^{1} \times \mathbb{P}^{1},\left(z, b_{1}, b_{2}\right)} \rightarrow T_{R \times R,\left(\left[\tilde{f}\left(C_{1}\right)\right],\left[\tilde{f}\left(C_{2}\right)\right]\right)} & \\
& =H^{0}\left(C_{1}, N_{\tilde{f}\left(C_{1}\right) / X}\right) \oplus H^{0}\left(C_{2}, N_{\tilde{f}\left(C_{2}\right) / X}\right)
\end{aligned}
$$

is at least $(r+m)$-dimensional. The desired result now follows from the following commutative diagram and the observation that the kernel of the bottom row is 2-dimensional:

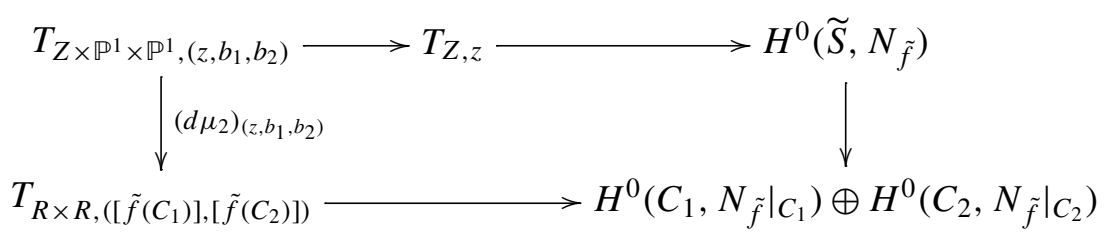

Proposition 2.1 will be enough for the proof of Theorem 1.1, but to prove Theorem 1.3 in the even case, we will need a slightly stronger variant. Let $f: Y \rightarrow X$ be a morphism between smooth varieties, and let $N_{f}$ be the normal sheaf of $f$

$$
0 \rightarrow T_{Y} \rightarrow f^{*} T_{X} \rightarrow N_{f} \rightarrow 0 .
$$

Suppose there is a dominant map $\pi: Y \rightarrow \mathbb{P}^{1}$, and let $M$ be the image of the map induced by $\pi$ on the tangent bundles $T_{Y} \rightarrow \pi^{*} T_{\mathbb{P} 1}$. Consider the push-out of the above sequence by the map $T_{Y} \rightarrow M$ :

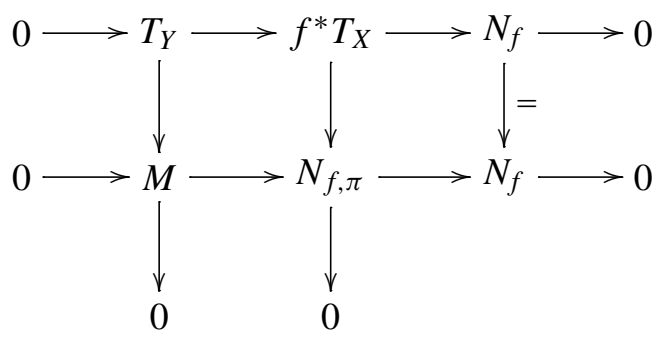

The sheaf $N_{f, \pi}$ in the above diagram will be referred to as the normal sheaf of $f$ relative to $\pi$. 
Property (ii) of Proposition 2.1 says that $H^{0}\left(S, N_{f}\right) \rightarrow H^{0}\left(C,\left.N_{f}\right|_{C}\right)$ is surjective. An argument parallel to the proof of Proposition 2.1 shows the following:

Proposition 2.2. Let $X$ be as in Proposition 2.1. Then property (ii) can be strengthened as follows:

(ii') If $N_{f}$ denotes the normal sheaf of $f$ and $N_{f, \pi}$ denotes the normal sheaf of $f$ relative to $\pi$, then the composition of the maps

$$
H^{0}\left(S, N_{f, \pi}\right) \rightarrow H^{0}\left(C,\left.N_{f, \pi}\right|_{C}\right) \rightarrow H^{0}\left(C,\left.N_{f}\right|_{C}\right)
$$

is surjective for a general fiber $C$ of $\pi$.

Moreover, if the general fibers of the MRC fibration of a desingularization of $R$ are at least $m$-dimensional, then there are $S$ and $f$ with properties (i) and (ii') such that the image of the map

$$
H^{0}\left(S, N_{f, \pi} \otimes I_{C}\right) \rightarrow H^{0}\left(C,\left.\left(N_{f} \otimes I_{C}\right)\right|_{C}\right)
$$

is at least $(m-1)$-dimensional.

\section{The case when $\frac{n+1}{2} \leq d$}

Let $X$ be a smooth hypersurface of degree $d$ in $\mathbb{P}^{n}$. Assume that a sweeping component $R$ of $R_{e}(X)$ is uniruled. The following result, along with Proposition 2.1, will prove Theorem 1.1.

Proposition 3.1. Suppose $d \leq n-3$, and let $S$ and $f$ be as in Proposition 2.1. If $C$ is a general fiber of $\pi: S \rightarrow \mathbb{P}^{1}$ and $I_{C}$ is the ideal sheaf of $C$ in $S$, then the restriction map

$$
H^{0}\left(S, f^{*} \mathcal{O}_{X}(2 d-n-1) \otimes I_{C}^{\vee}\right) \rightarrow H^{0}\left(C,\left.f^{*} \mathscr{O}_{X}(2 d-n-1) \otimes I_{C}^{\vee}\right|_{C}\right)
$$

is zero.

Proof of Theorem 1.1. Granting Proposition 3.1, since

$$
H^{0}\left(S, f^{*} \mathbb{O}_{X}(2 d-n-1) \otimes I_{C}^{\vee}\right) \rightarrow H^{0}\left(C,\left.f^{*} \mathscr{O}_{X}(2 d-n-1) \otimes I_{C}^{\vee}\right|_{C}\right)
$$

is the zero map, we have

$$
H^{0}\left(S, f^{*} \mathscr{O}_{X}(2 d-n-1)\right)=H^{0}\left(S, f^{*} \mathscr{O}_{X}(2 d-n-1) \otimes I_{C}^{\vee}\right) .
$$

Thus,

$$
\begin{aligned}
H^{0}\left(\mathbb{P}^{1}, \pi_{*} f^{*} \mathfrak{O}_{X}(2 d-n-1)\right) & =H^{0}\left(\mathbb{P}^{1}, \pi_{*}\left(f^{*} \mathscr{O}_{X}(2 d-n-1) \otimes I_{C}^{\vee}\right)\right) \\
& =H^{0}\left(\mathbb{P}^{1},\left(\pi_{*} f^{*} \mathscr{O}_{X}(2 d-n-1)\right) \otimes \mathbb{O}_{\mathbb{P}^{1}}(1)\right),
\end{aligned}
$$

which is only possible if $H^{0}\left(\mathbb{P}^{1}, \pi_{*} f^{*} \mathbb{O}_{X}(2 d-n-1)\right)$ vanishes. So we have $H^{0}\left(S, f^{*} \mathrm{O}_{X}(2 d-n-1)\right)=0$ and $d<(n+1) / 2$. 
Proof of Proposition 3.1.

Let $\omega_{S}$ be the canonical sheaf of $S$. By Serre duality and the long exact sequence of cohomology, it suffices to show that if $S$ and $f$ satisfy the properties of Proposition 2.1, then the restriction map

$$
H^{1}\left(S, f^{*} \mathbb{O}_{X}(n+1-2 d) \otimes \omega_{S}\right) \rightarrow H^{1}\left(C,\left.f^{*} \mathscr{O}_{X}(n+1-2 d) \otimes \omega_{S}\right|_{C}\right)
$$

is surjective. Let $N$ be the normal sheaf of the map $f: S \rightarrow X$, and let $N^{\prime}$ be the normal sheaf of the map $S \rightarrow \mathbb{P}^{n}$.

There is a short exact sequence

$$
0 \rightarrow N \rightarrow N^{\prime} \rightarrow f^{*} \mathrm{O}_{X}(d) \rightarrow 0 .
$$

Taking the $(n-3)$-rd exterior power of this sequence, we get the exact sequence

$$
0 \rightarrow \bigwedge^{n-3} N \otimes f^{*} \mathscr{O}_{X}(-d) \rightarrow \bigwedge^{n-3} N^{\prime} \otimes f^{*} \mathscr{O}_{X}(-d) \rightarrow \bigwedge^{n-4} N \rightarrow 0 .
$$

For an exact sequence of sheaves of $\mathrm{O}_{S}$-modules $0 \rightarrow E \rightarrow F \rightarrow M \rightarrow 0$ with $E$ and $F$ locally free of ranks $e$ and $f$, there is a natural map of sheaves

$$
\bigwedge^{f-e-1} M \otimes \bigwedge^{e} E \otimes\left(\bigwedge^{f} F\right)^{\vee} \rightarrow M^{\vee}
$$

which is defined locally at a point $s \in S$ as follows: assume $\gamma_{1}, \ldots, \gamma_{f-e-1} \in M_{s}$, $\alpha_{1}, \ldots, \alpha_{e} \in E_{s}$, and $\phi: \wedge^{f} F_{s} \rightarrow O_{S, s}$; then for $\gamma \in M_{s}$, we set $\gamma_{f-e}=\gamma$, and we define the map to be $\gamma \mapsto \phi\left(\tilde{\gamma}_{1} \wedge \tilde{\gamma}_{2} \wedge \cdots \wedge \tilde{\gamma}_{f-e} \wedge \alpha_{1} \wedge \cdots \wedge \alpha_{e}\right)$, where $\tilde{\gamma}_{i}$ is any lifting of $\gamma_{i}$ in $F_{s}$. Clearly, this map does not depend on the choice of the liftings, and thus it is defined globally. So from the short exact sequence $0 \rightarrow T_{S} \rightarrow f^{*} T_{X} \rightarrow N \rightarrow 0$, we get a map

$$
\wedge^{n-4} N \rightarrow N^{\vee} \otimes f^{*} \mathfrak{O}_{X}(n+1-d) \otimes \omega_{S},
$$

and from the short exact sequence $0 \rightarrow T_{S} \rightarrow f^{*} T_{\mathbb{P} n} \rightarrow N^{\prime} \rightarrow 0$, we get a map

$$
\bigwedge^{n-3} N^{\prime} \otimes f^{*} O_{X}(-d) \rightarrow\left(N^{\prime}\right)^{\vee} \otimes f^{*} O_{X}(n+1) \otimes \omega_{S}
$$

With the choices of the maps we have made, the following diagram, whose bottom row is obtained from dualizing sequence (1) and tensoring with

$$
f^{*} \mathbb{O}_{X}(n+1-2 d) \otimes \omega_{S}
$$

is commutative with exact rows:

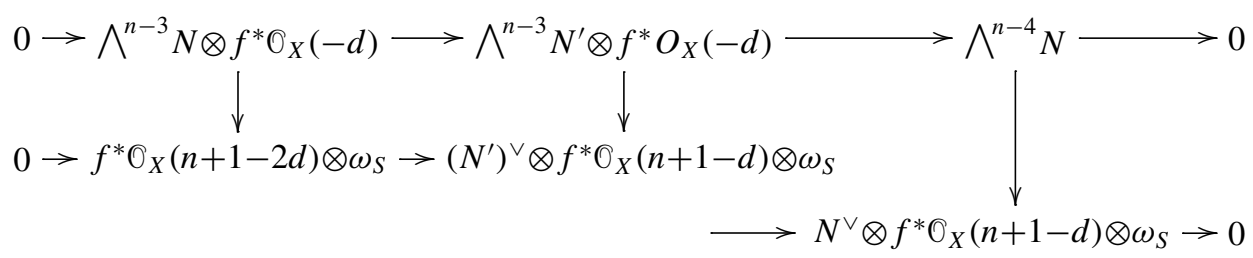


Since the cokernel of the first vertical map restricted to $C$ is a torsion sheaf, to show the assertion it suffices to show that the map

$$
H^{1}\left(S, \bigwedge^{n-3} N \otimes f^{*} \mathbb{O}_{X}(-d)\right) \rightarrow H^{1}\left(C,\left.\bigwedge^{n-3} N \otimes f^{*} \mathscr{O}_{X}(-d)\right|_{C}\right)
$$

is surjective. Applying the long exact sequence of cohomology to the top sequence, the surjectivity assertion follows if we show that

(1) $H^{0}\left(S, \bigwedge^{n-4} N\right) \rightarrow H^{0}\left(C,\left.\bigwedge^{n-4} N\right|_{C}\right)$ is surjective,

(2) $H^{1}\left(C,\left.\wedge^{n-3} N^{\prime} \otimes f^{*} \mathscr{O}_{X}(-d)\right|_{C}\right)=0$.

To prove (1), we consider the following commutative diagram:

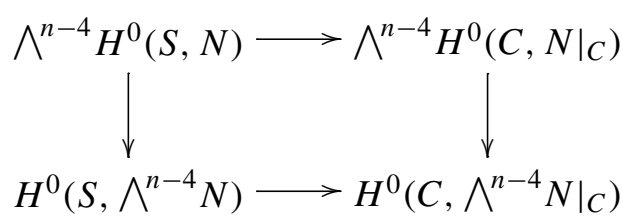

The top horizontal map is surjective since $H^{0}(S, N) \rightarrow H^{0}\left(C,\left.N\right|_{C}\right)$ is surjective, and the right vertical map is surjective since $\left.N\right|_{C}$ is a globally generated line bundle over $\mathbb{P}^{1}$. By commutativity of the diagram the bottom horizontal map is surjective.

To prove (2), note that there is a surjective map $f^{*} \mathscr{O}_{\mathbb{P}^{n}}(1)^{\oplus n+1} \rightarrow N^{\prime}$. Taking the $(n-3)$-rd exterior power, and then tensoring with $f^{*} O_{X}(-d)$, we get a surjective map

$$
f^{*} \mathcal{O}_{\mathbb{P}}(n-3-d)^{\oplus\left(\begin{array}{c}
n+1 \\
n-3
\end{array}\right)} \rightarrow \bigwedge^{n-3} N^{\prime} \otimes f^{*} \mathcal{O}_{X}(-d) .
$$

Restricting to $C$, since $n-3-d \geq 0$, we have

$$
H^{1}\left(C,\left.\bigwedge^{n-3} N^{\prime} \otimes f^{*} O_{X}(-d)\right|_{C}\right)=0 .
$$

Proof of Theorem 1.2. Suppose that $X$ is a smooth hypersurface of degree $n-2$ in $\mathbb{P}^{n}$. Let $C$ be a smooth rational curve of degree $e$ in $\mathbb{P}^{n}$ whose normal bundle $N_{C / \mathbb{P} n}$ is globally generated. If we write

$$
N_{C / \mathbb{P}^{n}}=\mathrm{O}_{C}\left(a_{1}\right) \oplus \cdots \oplus \mathcal{O}_{C}\left(a_{n-1}\right),
$$

then $\sum_{1 \leq i \leq n-1} a_{i}=e(n+1)-2$. Assume that $a_{i}+a_{j}<3 e$ for every $1 \leq i<j \leq n-1$.

Then $H^{1}\left(C,\left.\wedge^{n-3} N_{C / \mathbb{P}^{n}} \otimes \mathbb{O}_{\mathbb{P}^{n}}(-d)\right|_{C}\right)=0$, and so if $N^{\prime}$ is as in the proof of Theorem 1.1, then

$$
H^{1}\left(C,\left.\bigwedge^{n-3} N^{\prime} \otimes f^{*} \widehat{O}_{X}(-d)\right|_{C}\right)=0 .
$$

The assertion now follows from the proof of Theorem 1.1. 
We remark that when $d=n-1$ or $n$, the uniruledness of the sweeping subvarieties of $R_{e}(X)$ has been studied in [Beheshti and Starr 2008]. It is proved that if $e \leq n$, then a subvariety of $R_{e}(X)$ is nonuniruled if the curves parametrized by its points sweep out $X$ or a divisor in $X$.

\section{Cubic fourfolds}

In this section we prove Theorem 1.3. When $e \geq 5$ is odd, the theorem follows from Theorem 1.2 and [de Jong and Starr 2004, Proposition 7.1].

So let $e \geq 6$ be an even integer, and assume to the contrary that the general fibers of the MRC fibration of $R_{e}(X)$ are at least 2-dimensional. Let $S$ and $f$ be as in Proposition 2.2, and let $C$ be a general fiber of $\pi$. Set $N=N_{f}$ and $Q=N_{f, \pi}$. Then by Proposition 2.1 the following properties are satisfied:

- Property (i): The composition of the maps

$$
H^{0}(S, Q) \rightarrow H^{0}\left(S,\left.Q\right|_{C}\right) \rightarrow H^{0}\left(C,\left.N\right|_{C}\right)
$$

is surjective.

- Property (ii): The composition of the maps

$$
H^{0}\left(S, Q \otimes I_{C}\right) \rightarrow H^{0}\left(C,\left.Q \otimes I_{C}\right|_{C}\right) \rightarrow H^{0}\left(C,\left.N \otimes I_{C}\right|_{C}\right)
$$

is nonzero.

We show these lead to a contradiction. Note that $\left.I_{C}\right|_{C}$ is isomorphic to the trivial bundle $\mathrm{O}_{C}$, but we write $\left.I_{C}\right|_{C}$ instead of $\mathrm{O}_{C}$ to keep track of various maps and exact sequences involved in the proof.

Let $Q^{\prime}$ be the normal sheaf of the map $S \rightarrow \mathbb{P}^{5}$ relative to $\pi$. We have $\left.Q\right|_{C}=N_{C / X}$ and $\left.Q^{\prime}\right|_{C}=N_{C / \mathbb{P}^{5}}$. Since $N_{X / \mathbb{P}^{5}}=\mathscr{O}_{X}(3)$, there is a short exact sequence

$$
0 \rightarrow Q \rightarrow Q^{\prime} \rightarrow f^{*} \mathrm{O}_{X}(3) \rightarrow 0
$$

Taking exterior powers, we obtain the short exact sequence

$$
0 \rightarrow \bigwedge^{2} Q \otimes f^{*} \mathrm{O}_{X}(-3) \rightarrow \bigwedge^{2} Q^{\prime} \otimes f^{*} \mathscr{O}_{X}(-3) \rightarrow Q \rightarrow 0 .
$$

Since this sequence splits locally, its restriction to $C$ is also a short exact sequence

$$
\left.\left.\left.0 \rightarrow \bigwedge^{2} Q \otimes f^{*} \mathrm{O}_{X}(-3)\right|_{C} \rightarrow \bigwedge^{2} Q^{\prime} \otimes f^{*} \mathfrak{O}_{X}(-3)\right|_{C} \rightarrow Q\right|_{C} \rightarrow 0 .
$$

To get a contradiction, we show that the image of the boundary map

$$
\gamma: H^{0}\left(C,\left.Q\right|_{C}\right) \rightarrow H^{1}\left(C,\left.\wedge^{2} Q \otimes f^{*} \mathrm{O}_{X}(-3)\right|_{C}\right)
$$


is of codimension at least 2 in $H^{1}\left(C,\left.\wedge^{2} Q \otimes f^{*} \mathscr{O}_{X}(-3)\right|_{C}\right)$. This is not possible since by our assumption $N_{C / \mathbb{P}^{5}}=\mathscr{O}_{C}(3 e / 2)^{\oplus 2} \oplus \mathcal{O}_{C}((3 e / 2)-1)^{\oplus 2}$, and so

$$
\begin{aligned}
H^{1}\left(C,\left.\bigwedge^{2} Q^{\prime} \otimes f^{*} \mathscr{O}_{X}(-3)\right|_{C}\right) & =H^{1}\left(C,\left.\wedge^{2} N_{C / \mathbb{P}^{5}} \otimes f^{*} \mathscr{O}_{X}(-3)\right|_{C}\right) \\
& =H^{1}\left(C, \mathscr{O}_{C}(-2) \oplus \mathscr{O}_{C}(-1)^{\oplus 4} \oplus \mathscr{O}_{C}\right) \\
& =\mathbf{k} .
\end{aligned}
$$

Lemma 4.1. The kernel of the map $f^{*} T_{X} \rightarrow Q$ is a line bundle which contains $\bigwedge^{2} T_{S} \otimes \pi^{*} \omega_{\mathbb{P}^{1}}$ as a subsheaf.

Proof. The kernel of $f^{*} T_{X} \rightarrow Q$ is equal to the kernel of the map induced by $\pi$ on the tangent bundles $T_{S} \rightarrow \pi^{*} T_{\mathbb{P} 1}$, which we denote by $F$ :

$$
0 \rightarrow F \rightarrow T_{S} \rightarrow \pi^{*} T_{\mathbb{P} 1} .
$$

Since $F$ is reflexive, it is locally free on $S$, and it is clearly of rank 1 . Also, the composition of the maps

$$
\bigwedge^{2} T_{S} \otimes \pi^{*} \omega_{\mathbb{P} 1} \rightarrow \bigwedge^{2} T_{S} \otimes \Omega_{S}=T_{S} \rightarrow \pi^{*} T_{\mathbb{P} 1}
$$

is the zero map. So $\bigwedge^{2} T_{S} \otimes \pi^{*} \omega_{\mathbb{P}^{1}}$ is a subsheaf of $F$.

Given a section $r \in H^{0}\left(C,\left.Q \otimes I_{C}\right|_{C}\right)$, we can define a map

$$
\beta_{r}: H^{1}\left(C,\left.\bigwedge^{2} Q \otimes f^{*} \mathfrak{O}_{X}(-3)\right|_{C}\right) \longrightarrow H^{1}\left(C,\left.\omega_{S}\right|_{C}\right)=\mathbb{k}
$$

as follows. Let $F$ be the line bundle from the proof of Lemma 4.1. It follows from the proof of the lemma that there is an injection $\bigwedge^{2} T_{S} \otimes \pi^{*} \omega_{\mathbb{p}^{1}} \rightarrow F$, and from the short exact sequence

$$
0 \rightarrow F \rightarrow f^{*} T_{X} \rightarrow Q \rightarrow 0
$$

we get a generically injective map of sheaves

$$
\bigwedge^{3} Q \otimes F \rightarrow \bigwedge^{4} f^{*} T_{X}
$$

Combining these, we get a morphism

$$
\bigwedge^{3} Q \otimes\left(\omega_{S} \otimes \pi^{*} T_{\mathbb{P}^{1}}\right)^{\vee} \rightarrow \bigwedge^{4} f^{*} T_{X} .
$$

Since $\wedge^{4} f^{*} T_{X}=f^{*} \mathscr{O}_{X}(3)$, we get a generically injective map

$$
\Psi: \bigwedge^{3} Q \otimes f^{*} \mathrm{O}_{X}(-3) \otimes I_{C} \rightarrow \omega_{S} \otimes \pi^{*} T_{\mathbb{P}^{1}} \otimes I_{C},
$$

and by restricting to $C$, we get a map

$$
\left.\Psi\right|_{C}:\left.\left.\left(\bigwedge^{3} Q \otimes f^{*} O_{X}(-3) \otimes I_{C}\right)\right|_{C} \rightarrow \omega_{S}\right|_{C} .
$$


Finally, $r$ gives a map

$$
\Phi_{r}:\left.\left.\wedge^{2} Q \otimes f^{*} \mathscr{O}_{X}(-3)\right|_{C} \stackrel{\wedge r}{\rightarrow} \wedge^{3} Q \otimes f^{*} \mathscr{O}_{X}(-3) \otimes I_{C}\right|_{C}
$$

and we define $\beta_{r}$ to be the map induced by the composition $\left.\Psi\right|_{C} \circ \Phi_{r}$. Note that $\beta_{r}$ is nonzero if $r \neq 0$.

Lemma 4.2. For $r, r^{\prime} \in H^{0}\left(C,\left.Q \otimes I_{C}\right|_{C}\right), \operatorname{ker}\left(\beta_{r}\right)=\operatorname{ker}\left(\beta_{r^{\prime}}\right)$ if and only if $r$ and $r^{\prime}$ are scalar multiples of each other.

Proof. By Serre duality, it is enough to show that the images of the maps

$$
H^{0}\left(C,\left.I_{C}^{\vee}\right|_{C}\right)=H^{0}\left(C,\left.\omega_{S}^{\vee}\right|_{C} \otimes \omega_{C}\right) \underset{\beta_{r^{\prime}}}{\stackrel{\beta_{r}^{\vee}}{\longrightarrow}} H^{0}\left(C,\left.\left(\bigwedge^{2} Q^{\vee} \otimes f^{*} \mathfrak{O}_{X}(3)\right)\right|_{C} \otimes \omega_{C}\right)
$$

are the same if and only if $r$ and $r^{\prime}$ are scalar multiples of each other. Since $\left.Q\right|_{C}=N_{C / X}$, we have $\left.\wedge^{3} Q\right|_{C}=\bigwedge^{3} N_{C / X}=f^{*} O_{X}(3) \otimes \omega_{C}$, so

$$
\left.\left(\wedge^{2} Q^{\vee} \otimes f^{*} O_{X}(3)\right)\right|_{C} \otimes \omega_{C}=\left.Q\right|_{C},
$$

and the map

$$
\beta_{r}^{\vee}: H^{0}\left(C,\left.I_{C}^{\vee}\right|_{C}\right) \rightarrow H^{0}\left(C,\left.Q\right|_{C}\right)
$$

is simply given by $r$. Similarly, $\beta_{r^{\prime}}^{\vee}$ is given by $r^{\prime}$, and the lemma follows.

Recall that by definition, we have a short exact sequence

$$
\left.\left.\left.0 \rightarrow \pi^{*} T_{\mathbb{P}^{1}}\right|_{C} \rightarrow Q\right|_{C} \rightarrow N\right|_{C} \rightarrow 0,
$$

and $\left.\pi^{*} T_{\mathbb{P} 1}\right|_{C}=\left.I_{C}^{-1}\right|_{C}$. If we tensor this sequence with $\left.I_{C}\right|_{C}$, we get the short exact sequence

$$
\left.\left.0 \rightarrow \mathrm{O}_{C} \rightarrow Q \otimes I_{C}\right|_{C} \rightarrow N \otimes I_{C}\right|_{C} \rightarrow 0
$$

Let $i$ be a nonzero section in the image of $H^{0}\left(C, \mathscr{O}_{C}\right) \rightarrow H^{0}\left(C,\left.Q \otimes I_{C}\right|_{C}\right)$. Then $i$ induces a map

$$
\beta_{i}: H^{1}\left(C,\left.\bigwedge^{2} Q \otimes f^{*} \mathbb{O}_{X}(-3)\right|_{C}\right) \longrightarrow H^{1}\left(C,\left.\omega_{S}\right|_{C}\right)=\mathbb{k}
$$

as described before. Let

$$
\gamma: H^{0}\left(C,\left.Q\right|_{C}\right) \rightarrow H^{1}\left(C,\left.\bigwedge^{2} Q \otimes f^{*} \mathrm{O}_{X}(-3)\right|_{C}\right)
$$

be the connecting map in sequence (4).

Lemma 4.3. We have image $(\gamma) \subset \operatorname{ker} \beta_{i}$. 
Proof. Since the short exact sequence $0 \rightarrow N \rightarrow N^{\prime} \rightarrow f^{*} \mathbb{O}_{X}(3) \rightarrow 0$ splits locally, there is an exact sequence

$$
0 \rightarrow \bigwedge^{2} N \otimes f^{*} \mathrm{O}_{X}(-3) \rightarrow \bigwedge^{2} N^{\prime} \otimes f^{*} \mathscr{O}_{X}(-3) \rightarrow N \rightarrow 0 .
$$

Applying the long exact sequence of cohomology to the restriction of this sequence to $C$, we get a map

$$
H^{0}\left(C,\left.N\right|_{C}\right) \rightarrow H^{1}\left(C,\left.\wedge^{2} N \otimes f^{*} \mathrm{O}_{X}(-3)\right|_{C}\right) .
$$

Also from the exact sequence $0 \rightarrow T_{S} \rightarrow f^{*} T_{X} \rightarrow N \rightarrow 0$, we get a map

$$
\bigwedge^{2} T_{S} \otimes \bigwedge^{2} N \rightarrow \wedge^{4} f^{*} T_{X}=f^{*} O_{X}(3),
$$

and hence a map

$$
\bigwedge^{2} N \otimes f^{*} \mathscr{O}_{X}(-3) \rightarrow \omega_{S}
$$

It follows from the definition of $\beta_{i}$ that the map $\beta_{i} \circ \gamma$ factors through

$$
H^{0}\left(C,\left.Q\right|_{C}\right) \rightarrow H^{0}\left(C,\left.N\right|_{C}\right) \rightarrow H^{1}\left(C,\left.\bigwedge^{2} N \otimes f^{*} \mathfrak{O}_{X}(-3)\right|_{C}\right) \rightarrow H^{1}\left(C,\left.\omega_{S}\right|_{C}\right),
$$

so we have a commutative diagram

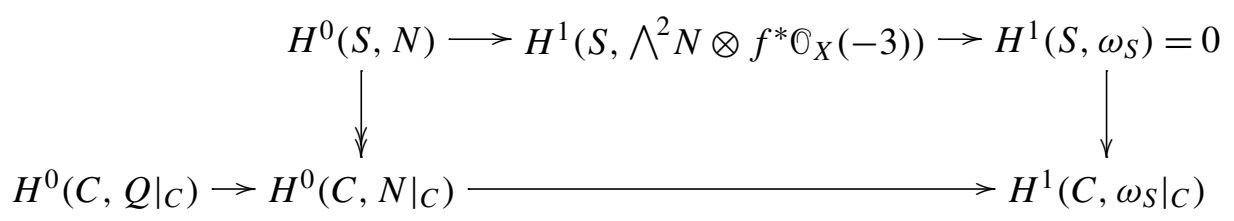

Thus we can conclude the assertion by the fact that the restriction map $H^{0}(S, N) \rightarrow$ $H^{0}\left(C,\left.N\right|_{C}\right)$ is surjective, and so the image of the composition of the above maps is contained in the image of the restriction map $H^{1}\left(S, \omega_{S}\right) \rightarrow H^{1}\left(C,\left.\omega_{S}\right|_{C}\right)$, which is zero.

In the following lemma we prove a similar result for the sections of $\left.Q \otimes I_{C}\right|_{C}$ which are obtained by restricting the global sections of $Q \otimes I_{C}$ to $C$.

Lemma 4.4. If $\tilde{r} \in H^{0}\left(S, Q \otimes I_{C}\right)$, and if $r=\left.\tilde{r}\right|_{C}$, then image $(\gamma) \subset \operatorname{ker}\left(\beta_{r}\right)$. Proof. We have a commutative diagram

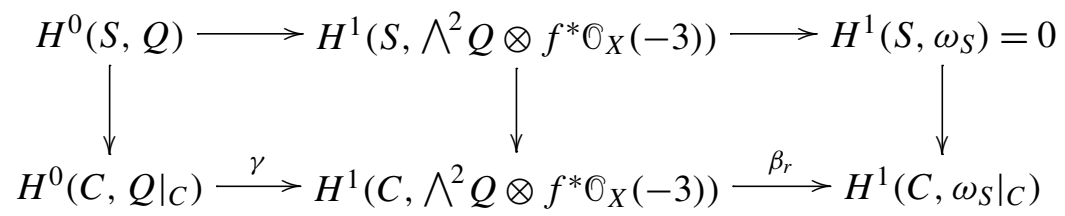

Therefore we have $\beta_{r}(\gamma(u))=0$ for any $u \in H^{0}\left(C,\left.Q\right|_{C}\right)$ in the image of the 
restriction map $H^{0}(S, Q) \rightarrow H^{0}\left(C,\left.Q\right|_{C}\right)$. Consider the exact sequence

$$
\left.\left.\left.0 \rightarrow I_{C}^{-1}\right|_{C} \rightarrow Q\right|_{C} \rightarrow N\right|_{C} \rightarrow 0 .
$$

From the hypothesis that the composition map

$$
H^{0}(S, Q) \rightarrow H^{0}\left(C,\left.Q\right|_{C}\right) \rightarrow H^{0}\left(C,\left.N\right|_{C}\right)
$$

is surjective, we see that to prove the statement it is enough to show that for any nonzero $u$ in the image of $H^{0}\left(C,\left.I_{C}^{-1}\right|_{C}\right) \rightarrow H^{0}\left(C,\left.Q\right|_{C}\right)$, we have $\gamma(u) \in \operatorname{ker} \beta_{r}$.

Consider the following diagram, where $\lambda$ is obtained from applying the long exact sequence of cohomology to the third wedge power of sequence (2), and $\psi$ is induced by the map $\left.\Psi\right|_{C}$ :

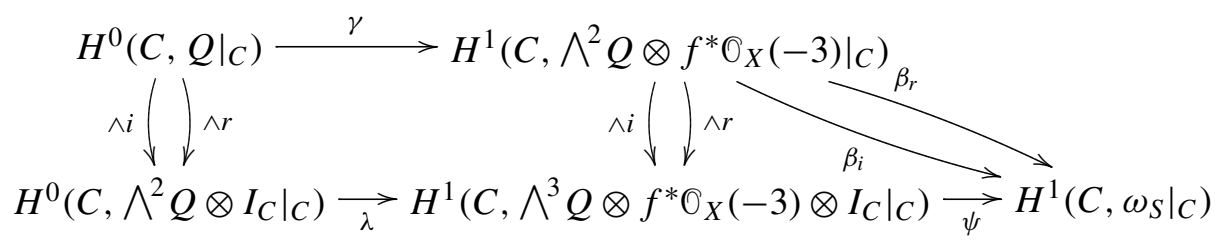

Then we have

$$
\begin{aligned}
\beta_{r} \circ \gamma(u) & =\psi \circ \lambda(u \wedge r) \\
& =\psi \circ \lambda(r \wedge i) \quad \text { (up to a scalar factor) } \\
& =\beta_{i} \circ \gamma(r) \\
& =0,
\end{aligned}
$$

where the last equality comes from $\gamma\left(H^{0}\left(C,\left.Q\right|_{C}\right)\right) \subset \operatorname{ker} \beta_{i}$, by Lemma 4.3.

Now, let $\tilde{r}_{0} \in H^{0}\left(S, Q \otimes I_{C}\right)$ be so that its image in $H^{0}\left(C,\left.N \otimes I_{C}\right|_{C}\right)$ is nonzero. Such an $\tilde{r}_{0}$ exists by Property (ii). Then $r_{0}:=\left.\tilde{r}_{0}\right|_{C}$ defines a map $\beta_{r_{0}}$. Since the image of $r_{0}$ in $H^{0}\left(C,\left.N \otimes I_{C}\right|_{C}\right)$ is nonzero, $r_{0}$ and $i$ are not scalar multiples, so according to Lemma $4.2, \operatorname{ker} \beta_{r_{0}} \neq \operatorname{ker} \beta_{i}$. Thus the codimension of $\operatorname{ker} \beta_{i} \cap \operatorname{ker} \beta_{r_{0}}$ is at least 2. On the other hand, by the previous lemmas, image $(\gamma) \subset \operatorname{ker} \beta_{i} \cap \operatorname{ker} \beta_{r_{0}}$. This is a contradiction since $\operatorname{dim} H^{1}\left(C,\left.\wedge^{2} Q^{\prime} \otimes f^{*} \mathscr{O}_{X}(-3)\right|_{C}\right)=1$.

\section{The case when $d<\frac{n+1}{2}$}

Throughout this section, $X \subset \mathbb{P}^{n}$ is a general hypersurface of degree $d<(n+1) / 2$. By the main theorem of [Harris et al. 2004], $R_{e}(X)$ is irreducible for every $e \geq 1$. If $d^{2} \leq n$ and $e \geq 2$, then by [de Jong and Starr 2006; Starr 2006], the space of rational curves of degree $e$ in $X$ passing through two general points of $X$ is rationally connected. In particular, $R_{e}(X)$ is rationally connected for $e \geq 2$. If $e=1$, then $R_{1}(X)$ is the Fano variety of lines in $X$ which is rationally connected if and only if 
$d^{2}+d \leq 2 n$ [Kollár 1996, V.4.7]. In this section, we will consider the case when $d^{2}+d>2 n$.

Assume that $R_{e}(X)$ is uniruled. Then there are $S$ and $f$ with the two properties given in Proposition 2.1. We can take the pair $(S, f)$ to be minimal in the sense that a component of a fiber of $\pi$ which is contracted by $f$ cannot be blown down. Let $N$ be the normal sheaf of $f$, and let $C$ be a general fiber of $\pi$ with ideal sheaf $I_{C}$ in $S$. Denote by $H$ the pullback of a hyperplane in $\mathbb{P}^{n}$ to $S$, and denote by $K$ a canonical divisor on $S$. From the exact sequences $0 \rightarrow T_{S} \rightarrow f^{*} T_{X} \rightarrow N \rightarrow 0$ and $0 \rightarrow f^{*} T_{X} \rightarrow f^{*} T_{\mathbb{P} n} \rightarrow f^{*} \mathrm{O}_{\mathbb{p} n}(d) \rightarrow 0$, we get

$\chi\left(N \otimes I_{C}\right)$

$$
\begin{gathered}
=(n+1) \chi\left(f^{*} \mathbb{O}_{\mathbb{P}^{n}}(1) \otimes I_{C}\right)-\chi\left(f^{*} \mathbb{O}_{\mathbb{P}^{n}}(d) \otimes I_{C}\right)-\chi\left(I_{C}\right)-\chi\left(T_{S} \otimes I_{C}\right) \\
=(n+1)\left(\frac{(H-C) \cdot(H-C-K)}{2}+1\right)-\frac{(d H-C) \cdot(d H-C-K)}{2}-1 \\
\quad-\frac{-C \cdot(-C-K)}{2}-1-\left(2 K^{2}-14\right) \\
=\frac{\left(n+1-d^{2}\right)}{2} H^{2}-\frac{(n+1-d)}{2} H \cdot K-2 K^{2}-(n+1-d) e+14 .
\end{gathered}
$$

We claim that $2 \mathrm{H}+2 \mathrm{C}+\mathrm{K}$ is base-point free and hence has a nonnegative self-intersection number. By the main theorem of [Reider 1988], if $2 H+2 C+K$ is not base-point free, then there exists an effective divisor $E$ such that either

$$
(2 H+2 C) \cdot E=1, E^{2}=0 \quad \text { or } \quad(2 H+2 C) \cdot E=0, E^{2}=-1 .
$$

The first case is clearly not possible. In the second case, $H \cdot E=0$, and $C \cdot E=0$. So $E$ is a component of one of the fibers of $\pi$ which is contracted by $f$ and which is a $(-1)$-curve. This contradicts the assumption that $(S, f)$ is minimal. Thus $(2 H+2 C+K)^{2} \geq 0$. Also, since $H^{1}\left(S, f^{*} O_{X}(-1)\right)=0$, we have

$$
H \cdot(H+K)=2 \chi\left(f^{*} \mathscr{O}_{X}(-1)\right)-2 \geq-2,
$$

so we can write

$$
\begin{aligned}
\chi\left(N \otimes I_{C}\right) & =\frac{2 n+2-d^{2}-d}{2} H^{2}-(n-d-15)(e-1)-2-2(2 H+2 C+K)^{2} \\
& -\frac{n-d-15}{2}(H \cdot(H+K)+2) \\
& \leq \frac{2 n+2-d^{2}-d}{2} H^{2}-(n-d-15)(e-1)-2,
\end{aligned}
$$

and therefore $\chi\left(N \otimes I_{C}\right)$ is negative when $d^{2}+d \geq 2 n+2$ and $n \geq 30$.

The Leray spectral sequence gives a short exact sequence

$$
0 \rightarrow H^{1}\left(\mathbb{P}^{1}, \pi_{*}\left(N \otimes I_{C}\right)\right) \rightarrow H^{1}\left(S, N \otimes I_{C}\right) \rightarrow H^{0}\left(\mathbb{P}^{1}, R^{1} \pi_{*}\left(N \otimes I_{C}\right)\right) \rightarrow 0,
$$


and by our assumption on $S$ and $f, H^{1}\left(\mathbb{P}^{1}, \pi_{*}\left(N \otimes I_{C}\right)\right)=0$. If we could choose $S$ such that $H^{0}\left(\mathbb{P}^{1}, R^{1} \pi_{*}\left(N \otimes I_{C}\right)\right)=0$, then we could conclude that $\chi\left(N \otimes I_{C}\right) \geq 0$, and hence $R_{e}(X)$ could not be uniruled for $d^{2}+d \geq 2 n+2$ and $n \geq 30$.

We cannot show that for a general $X$, a minimal pair $(S, f)$ as in Proposition 2.1 can be chosen so that $H^{0}\left(\mathbb{P}^{1}, R^{1} \pi_{*}\left(N \otimes I_{C}\right)\right)=0$. However, we prove that if $X$ is general and $(S, f)$ is minimal, then for every $t \geq 1$,

$$
H^{0}\left(\mathbb{P}^{1}, R^{1} \pi_{*}\left(N \otimes I_{C} \otimes f^{*} \mathcal{O}_{X}(t)\right)\right)=0 .
$$

We also show that if $t \geq 0$ and $f(C)$ is $t$-normal, then

$$
H^{1}\left(\mathbb{P}^{1}, \pi_{*}\left(N \otimes I_{C} \otimes f^{*} \mathscr{O}_{X}(t)\right)\right)=0 .
$$

These imply that $\chi\left(N \otimes I_{C} \otimes f^{*} O_{X}(t)\right)$ is nonnegative when $X$ is general and $f(C)$ is $t$-normal. To finish the proof of Theorem 1.4, we compute $\chi\left(N \otimes I_{C} \otimes f^{*} \mathbb{O}_{X}(t)\right)$ directly and show that it is negative when the inequality in the statement of the theorem holds.

Proof of Theorem 1.4. Let $X$ be a general hypersurface of degree $d$ in $\mathbb{P}^{n}$. If $R_{e}(X)$ is uniruled, then there are $S$ and $f$ as in Proposition 2.1. Assume the pair $(S, f)$ is minimal. Let $N$ be the normal sheaf of $f$, and let $C$ be a general fiber of $\pi$. Then $H^{0}(S, N) \rightarrow H^{0}\left(C,\left.N\right|_{C}\right)$ is surjective. The restriction map $H^{0}\left(S, f^{*} \mathrm{O}_{X}(m)\right) \rightarrow$ $H^{0}\left(C,\left.f^{*} \mathrm{O}_{X}(m)\right|_{C}\right)$ is also surjective since $f(C)$ is $m$-normal, so the restriction map $H^{0}\left(S, N \otimes f^{*} \mathscr{O}_{X}(m)\right) \rightarrow H^{0}\left(C,\left.N \otimes f^{*} \mathscr{O}_{X}(m)\right|_{C}\right)$ is surjective as well. Therefore,

$$
H^{1}\left(\mathbb{P}^{1}, \pi_{*}\left(N \otimes f^{*} \mathscr{O}_{X}(m) \otimes I_{C}\right)\right)=0 .
$$

Now let $C$ be an arbitrary fiber of $\pi$, and let $C^{0}$ be an irreducible component of $C$. Then by Proposition 5.2, $\left.f^{*}\left(T_{X}(t)\right)\right|_{C^{0}}$ is globally generated for every $t \geq 1$, and hence $\left.N \otimes f^{*} \mathscr{O}_{X}(t)\right|_{C^{0}}$ is globally generated too. So Lemma 5.1 shows that for every $t \geq 1$,

$$
H^{0}\left(\mathbb{P}^{1}, R^{1} \pi_{*}\left(N \otimes f^{*} \mathbb{O}_{X}(t) \otimes I_{C}\right)\right)=0 .
$$

By the Leray spectral sequence,

$$
\begin{aligned}
H^{1}(S, & \left.N \otimes f^{*} \mathscr{O}_{X}(m) \otimes I_{C}\right) \\
& =H^{1}\left(\mathbb{P}^{1}, \pi_{*}\left(N \otimes f^{*} \mathscr{O}_{X}(m) \otimes I_{C}\right)\right) \oplus H^{0}\left(\mathbb{P}^{1}, R^{1} \pi_{*}\left(N \otimes f^{*} \mathscr{O}_{X}(m) \otimes I_{C}\right)\right) \\
& =0,
\end{aligned}
$$

and therefore, $\chi\left(N \otimes f^{*} \mathscr{O}_{X}(m) \otimes I_{C}\right) \geq 0$. We next compute $\chi\left(N \otimes f^{*} \mathscr{O}_{X}(m) \otimes I_{C}\right)$. For an integer $t \geq 0$, set

$$
a_{t}=\chi\left(N \otimes I_{C} \otimes f^{*} O_{X}(t)\right) .
$$


We have

$$
a_{t}=\chi\left(N \otimes I_{C}\right)+\frac{2 t(n+1-d)+t^{2}(n-3)}{2} H^{2}-\frac{t(n-5)}{2} H \cdot K-t(n-3) e .
$$

So

$$
a_{t}=\frac{b_{t}}{2} H^{2}+\frac{c_{t}}{2} H \cdot K-2 K^{2}+d_{t},
$$

where

$$
\begin{aligned}
b_{t} & =\left(n+1-d^{2}\right)+2 t(n+1-d)+t^{2}(n-3), \\
c_{t} & =-(n+1-d)-t(n-5), \\
\text { and } \quad d_{t} & =-t(n-3) e-(n+1-d) e+14 .
\end{aligned}
$$

A computation similar to the computation in the beginning of this section shows that

$$
\begin{aligned}
a_{t}= & \frac{b_{t}-c_{t}}{2} H^{2}-2(2 H+2 C+K)^{2}+\frac{c_{t}+16}{2}(H \cdot(H+K)+2) \\
& +\left(d_{t}-c_{t}-32+16 e\right)
\end{aligned}
$$

Since $d_{t}-c_{t}-32+16 e=-(e-1)(n-15-d+t(n-3))-2 t-2$, and since $n-15-d+t(n-3) \geq 2 n-d-18 \geq 0$ for $t \geq 1$ and $n \geq 12$, we get

$$
a_{t}<\frac{b_{t}-c_{t}}{2} H^{2}
$$

When $d^{2}+(2 t+1) d \geq(t+1)(t+2) n+2, b_{t}<c_{t}$, and so $a_{t}<0$. If we let $t=m$, we get the desired result.

Lemma 5.1. If $E$ is a locally free sheaf on $S$ such that for every irreducible component $C^{0}$ of a fiber of $\pi,\left.E\right|_{C^{0}}$ is globally generated, then $R^{1} \pi_{*} E=0$.

Proof. By cohomology and base change [Hartshorne 1977, Theorem III.12.11], it suffices to prove that for every fiber $C$ of $\pi, H^{1}\left(C,\left.E\right|_{C}\right)=0$. We first show that if $l$ is the number of irreducible components of $C$ counted with multiplicity, then we can write $C=C_{1}+\cdots+C_{l}$ such that each $C_{i}$ is an irreducible component of $C$ and for every $1 \leq i \leq l-1,\left(C_{1}+\cdots+C_{i}\right) \cdot C_{i+1} \leq 1$. This is proven by induction on $l$. If $l=1$, there is nothing to prove. Otherwise, there is at least one component $C^{0}$ of $C$ which can be contracted. Let $r$ be the multiplicity of $C^{0}$ in $C$. Blowing down $C^{0}$, we get a rational surface $S^{\prime}$ over $\mathbb{P}^{1}$. Denote by $C^{\prime}$ the blow-down of $C$. Then by the induction hypothesis, we can write

$$
C^{\prime}=C_{1}^{\prime}+\cdots+C_{l-r}^{\prime}
$$


such that $\left(C_{1}^{\prime}+\cdots+C_{i}^{\prime}\right) \cdot C_{i+1}^{\prime} \leq 1$ for every $1 \leq i \leq l-r-1$. Let $C_{i}$ be the proper transform of $C_{i}^{\prime}$. Then if in the above sum we replace $C_{i}^{\prime}$ by $C_{i}$ when $C_{i}$ does not intersect $C^{0}$, and by $C_{i}+C^{0}$ when $C_{i}$ intersects $C^{0}$, we get the desired result for $C$.

Since $\left.E\right|_{C_{i+1}}$ is globally generated, $H^{1}\left(C_{i+1},\left.E\left(-C_{1}-\cdots-C_{i}\right)\right|_{C_{i+1}}\right)=0$ for every $0 \leq i \leq l-1$. On the other hand, for every $0 \leq i \leq l-2$, we have a short exact sequence of $\mathrm{O}_{S}$-modules

$$
\begin{aligned}
\left.0 \rightarrow E\left(-C_{1}-\cdots-C_{i+1}\right)\right|_{C_{i+2}+\cdots+C_{l}} \rightarrow E\left(-C_{1}\right. & \left.-\cdots-C_{i}\right)\left.\right|_{C_{i+1}+\cdots+C_{l}} \\
& \left.\rightarrow E\left(-C_{1}-\cdots-C_{i}\right)\right|_{C_{i+1}} \rightarrow 0 .
\end{aligned}
$$

So a decreasing induction on $i$ shows that for every $0 \leq i \leq l-2$, we have $H^{1}\left(S,\left.E\left(-C_{1}-\cdots-C_{i}\right)\right|_{C_{i+1}+\cdots+C_{l}}\right)=0$. Letting $i=0$, the statement follows.

Proposition 5.2. Let $X \subset \mathbb{P}^{n}$ be a general hypersurface of degree $d$.

(i) For any morphism $h: \mathbb{P}^{1} \rightarrow X, h^{*}\left(T_{X}(1)\right)$ is globally generated.

(ii) If $C$ is a smooth, rational, $d$-normal curve on $X$, then $H^{1}\left(C,\left.T_{X}\right|_{C}\right)=0$.

Proof. (i) This follows from [Voisin 1996, Proposition 1.1]. We give a proof here for the sake of completeness. Consider the short exact sequence

$$
0 \rightarrow h^{*} T_{X} \rightarrow h^{*} T_{\mathbb{P}^{n}} \rightarrow h^{*} \mathrm{O}_{X}(d) \rightarrow 0 .
$$

Since $X$ is general, the image of the pullback map

$$
H^{0}\left(X, \mathrm{O}_{X}(d)\right) \rightarrow H^{0}\left(\mathbb{P}^{1}, h^{*} \mathrm{O}_{X}(d)\right)
$$

is contained in the image of the map $H^{0}\left(\mathbb{P}^{1}, h^{*} T_{\mathbb{P} n}\right) \rightarrow H^{0}\left(\mathbb{P}^{1}, h^{*} \widehat{O}_{X}(d)\right)$. Choose a homogeneous coordinate system for $\mathbb{P}^{n}$. Let $p$ be a point in $\mathbb{P}^{1}$, and without loss of generality assume that $h(p)=(1: 0: \cdots: 0)$. We show that for any $\left.r \in h^{*}\left(T_{X}(1)\right)\right|_{p}$, there is $\tilde{r} \in H^{0}\left(\mathbb{P}^{1}, h^{*}\left(T_{X}(1)\right)\right)$ such that $\left.\tilde{r}\right|_{p}=r$.

Consider the exact sequence

$$
0 \longrightarrow H^{0}\left(\mathbb{P}^{1}, h^{*} T_{X}(1)\right) \longrightarrow H^{0}\left(\mathbb{P}^{1}, h^{*} T_{\mathbb{P}^{n}}(1)\right) \stackrel{\phi}{\longrightarrow} H^{0}\left(\mathbb{P}^{1}, h^{*} \mathrm{O}_{X}(d+1)\right) .
$$

Denote by $s$ the image of $r$ in $\left.h^{*}\left(T_{\mathbb{P}^{n}}(1)\right)\right|_{p}$. There exists $S \in H^{0}\left(\mathbb{P}^{n}, T_{\mathbb{P} n}(1)\right)$ such that the restriction of $\tilde{s}:=h^{*}(S)$ to $p$ is $s$. Denote by $T$ the image of $S$ in $H^{0}\left(\mathbb{P}^{n}, \mathcal{O}_{\mathbb{P}^{n}}(d+1)\right)$, and let $\tilde{t}=h^{*}(T)$. Then $T$ is a form of degree $d+1$ on $\mathbb{P}^{n}$, and since $\left.\tilde{t}\right|_{p}=0$, we can write $T=x_{1} G_{1}+\cdots+x_{n} G_{n}$, where the $G_{i}$ are forms of degree $d$. Our assumption implies that for every $1 \leq i \leq n$, there is $\tilde{s}_{i} \in H^{0}\left(\mathbb{P}^{1}, h^{*} T_{\mathbb{P} n}\right)$ such that $\phi\left(\tilde{s}_{i}\right)=h^{*} G_{i}$. Then

$$
\phi\left(\tilde{s}-h^{*}\left(x_{1}\right) \tilde{s}_{1}-\cdots-h^{*}\left(x_{n}\right) \tilde{s}_{n}\right)=\tilde{t}-h^{*}\left(x_{1} G_{1}\right)-\cdots-h^{*}\left(x_{n} G_{n}\right)=0,
$$

and therefore there is some $\tilde{r} \in H^{0}\left(\mathbb{P}^{1}, h^{*}\left(T_{X}(1)\right)\right)$ whose image is

$$
\tilde{s}-h^{*}\left(x_{1}\right) \tilde{s}_{1}-\cdots-h^{*}\left(x_{n}\right) \tilde{s}_{n} .
$$


Since $\left.\left(\tilde{s}-h^{*}\left(x_{1}\right) \tilde{s}_{1}-\cdots-h^{*}\left(x_{n}\right) \tilde{s}_{n}\right)\right|_{p}=\left.\tilde{s}\right|_{p}=s$, we have $\left.\tilde{r}\right|_{p}=r$.

(ii) There is a short exact sequence

$$
\left.\left.0 \rightarrow T_{X}\right|_{C} \rightarrow T_{\mathbb{P} n}\right|_{C} \rightarrow \mathrm{O}_{C}(d) \rightarrow 0 .
$$

The fact that $X$ is general implies that any section of $O_{C}(d)$ ) which is the restriction of a section of $\mathscr{O}_{\mathbb{P}}(d)$ can be lifted to a section of $\left.T_{\mathbb{P}^{n}}\right|_{C}$. Since the first cohomology group of $\left.T_{\mathbb{P} n}\right|_{C}$ vanishes, the result follows.

Although for every $e$ and $n$ with $e \geq n+1 \geq 4$, there are smooth nondegenerate rational curves of degree $e$ in $\mathbb{P}^{n}$ which are not $(e-n)$-normal [Gruson et al. 1983, Theorem 3.1], a general smooth rational curve of degree $e$ in a general hypersurface of degree $d$ has possibly a much smaller normality: if a maximal-rank type conjecture holds for rational curves contained in general hypersurfaces (at least when $d<(n+1) / 2)$, then it follows that if $c$ is the smallest positive number such that $\left(\begin{array}{c}n+c \\ n\end{array}\right)-\left(\begin{array}{c}n+c-d \\ n\end{array}\right) \geq c e+1$, a general smooth rational curve of degree $e$ in a general hypersurface of degree $d$ in $\mathbb{P}^{n}$ is $c$-normal.

\section{Acknowledgments}

I am grateful to Izzet Coskun, N. Mohan Kumar, Mike Roth, and Jason Starr for many helpful conversations. I also thank the referee for a careful reading of the paper and several significant suggestions.

\section{References}

[Beheshti and Starr 2008] R. Beheshti and J. M. Starr, "Rational surfaces in index-one Fano hypersurfaces”, J. Algebraic Geom. 17:2 (2008), 255-274. MR 2008j:14075 Zbl 1141.14024

[Coskun and Starr 2009] I. Coskun and J. Starr, "Rational curves on smooth cubic hypersurfaces", Int. Math. Res. Not. 2009:24 (2009), 4626-4641. MR 2010j:14056 Zbl 1200.14051

[Gruson et al. 1983] L. Gruson, R. Lazarsfeld, and C. Peskine, "On a theorem of Castelnuovo, and the equations defining space curves", Invent. Math. 72:3 (1983), 491-506. MR 85g:14033 Zbl 0565.14014

[Harris et al. 2004] J. Harris, M. Roth, and J. Starr, "Rational curves on hypersurfaces of low degree", J. Reine Angew. Math. 571 (2004), 73-106. MR 2005e:14067 Zbl 1052.14027

[Hartshorne 1977] R. Hartshorne, Algebraic geometry, Graduate Texts in Mathematics 52, Springer, New York, 1977. MR 57 \#3116 Zbl 0367.14001

[de Jong and Starr 2004] A. J. de Jong and J. Starr, "Cubic fourfolds and spaces of rational curves", Illinois J. Math. 48:2 (2004), 415-450. MR 2006e:14007 Zbl 1081.14007

[de Jong and Starr 2006] A. de Jong and J. Starr, "Low degree complete intersections are rationally simply connected", preprint, 2006, available at http://www.math.sunysb.edu/ jstarr/papers/ nk1006g.pdf.

[Kollár 1996] J. Kollár, Rational curves on algebraic varieties, Ergeb. Math. Grenzgeb. (3) 32, Springer, Berlin, 1996. MR 98c:14001 Zbl 0877.14012 
[Kollár et al. 1992] J. Kollár, Y. Miyaoka, and S. Mori, "Rational connectedness and boundedness of Fano manifolds", J. Differential Geom. 36:3 (1992), 765-779. MR 94g:14021 Zbl 0759.14032

[Reider 1988] I. Reider, "Vector bundles of rank 2 and linear systems on algebraic surfaces", Ann. of Math. (2) 127:2 (1988), 309-316. MR 89e:14038 Zbl 0663.14010

[Starr 2003] J. Starr, "The Kodaira dimension of spaces of rational curves on low degree hypersurfaces", preprint, 2003. arXiv math/0305432

[Starr 2006] J. Starr, "Hypersurfaces of low degree are rationally 1-connected", preprint, 2006. arXiv math/0602641

[Voisin 1996] C. Voisin, "On a conjecture of Clemens on rational curves on hypersurfaces", $J$. Differential Geom. 44:1 (1996), 200-213. MR 97j:14047 Zbl 0883.14022

Communicated by David Eisenbud

Received 2010-09-20 Revised 2011-06-19 Accepted 2011-07-28

beheshti@math.wustl.edu Department of Mathematics, Washington University, Campus Box 1146, Saint Louis, MO 63130, United States 


\section{Algebra \& Number Theory}

msp.berkeley.edu/ant

\section{EDITORS}

MANAGING EDITOR

Bjorn Poonen

Massachusetts Institute of Technology

Cambridge, USA

\author{
EDITORIAL BOARD CHAIR \\ David Eisenbud \\ University of California \\ Berkeley, USA
}

\section{BOARD OF EDITORS}

Georgia Benkart

Dave Benson

Richard E. Borcherds

John H. Coates

J-L. Colliot-Thélène

Brian D. Conrad

Hélène Esnault

Hubert Flenner

Edward Frenkel

Andrew Granville

Joseph Gubeladze

Ehud Hrushovski

Craig Huneke

Mikhail Kapranov

Yujiro Kawamata

János Kollár

Yuri Manin

Barry Mazur

Philippe Michel

Susan Montgomery
University of Wisconsin, Madison, USA

University of Aberdeen, Scotland

University of California, Berkeley, USA

University of Cambridge, UK

CNRS, Université Paris-Sud, France

University of Michigan, USA

Universität Duisburg-Essen, Germany

Ruhr-Universität, Germany

University of California, Berkeley, USA

Université de Montréal, Canada

San Francisco State University, USA

Hebrew University, Israel

University of Kansas, USA

Yale University, USA

University of Tokyo, Japan

Princeton University, USA

Northwestern University, USA

Harvard University, USA

École Polytechnique Fédérale de Lausanne

University of Southern California, USA
Shigefumi Mori

Raman Parimala

Jonathan Pila

Victor Reiner

Karl Rubin

Peter Sarnak

Joseph H. Silverman

Michael Singer

Ronald Solomon

Vasudevan Srinivas

J. Toby Stafford

Bernd Sturmfels

Richard Taylor

Ravi Vakil

Michel van den Bergh

Marie-France Vignéras

Kei-Ichi Watanabe

Andrei Zelevinsky

Efim Zelmanov
RIMS, Kyoto University, Japan

Emory University, USA

University of Oxford, UK

University of Minnesota, USA

University of California, Irvine, USA

Princeton University, USA

Brown University, USA

North Carolina State University, USA

Ohio State University, USA

Tata Inst. of Fund. Research, India

University of Michigan, USA

University of California, Berkeley, USA

Harvard University, USA

Stanford University, USA

Hasselt University, Belgium

Université Paris VII, France

Nihon University, Japan

Northeastern University, USA

University of California, San Diego, USA

\section{PRODUCTION}

contact@msp.org

Silvio Levy, Scientific Editor

See inside back cover or www.jant.org for submission instructions.

The subscription price for 2012 is US \$175/year for the electronic version, and \$275/year (+\$40 shipping outside the US) for print and electronic. Subscriptions, requests for back issues from the last three years and changes of subscribers address should be sent to Mathematical Sciences Publishers, Department of Mathematics, University of California, Berkeley, CA 94720-3840, USA.

Algebra \& Number Theory (ISSN 1937-0652) at Mathematical Sciences Publishers, Department of Mathematics, University of California, Berkeley, CA 94720-3840 is published continuously online. Periodical rate postage paid at Berkeley, CA 94704, and additional mailing offices.

ANT peer review and production are managed by EditFLOW ${ }^{\circledR}$ from Mathematical Sciences Publishers.

PUBLISHED BY

mathematical sciences publishers

http://msp.org/

A NON-PROFIT CORPORATION

Typeset in IAT $_{\mathrm{E}} \mathrm{X}$

Copyright (C2012 by Mathematical Sciences Publishers 


\section{Algebra \& Number Theory}

Volume $6 \quad$ No. $4 \quad 2012$

Spherical varieties and integral representations of $L$-functions

YIANNIS SAKELLARIDIS

Nonuniruledness results for spaces of rational curves in hypersurfaces

ROYA BEHESHTI

Degeneracy of triality-symmetric morphisms

DAVE ANDERSON

Multi-Frey $\mathbb{Q}$-curves and the Diophantine equation $a^{2}+b^{6}=c^{n}$

MiChaEl A. BENNETT and IMIN CHEN

Detaching embedded points

DAWEI CHEN and SCOTT NOLLET

Moduli of Galois $p$-covers in mixed characteristics

DAN ABRAMOVICH and MATTHIEU ROMAGNY

Block components of the Lie module for the symmetric group

ROGER M. BRYANT and KARIN ERDMANN

Basepoint-free theorems: saturation, b-divisors, and canonical bundle formula

OSAMU FUJINO

Realizing large gaps in cohomology for symmetric group modules

DAVID J. HEMMER 